\title{
Attentional capture under high perceptual load
}

\author{
Joshua D. Cosman and Shaun P. Vecera \\ University of Iowa, Iowa City, Iowa
}

\begin{abstract}
Attentional capture by abrupt onsets can be modulated by several factors, including the complexity, or perceptual load, of a scene. We have recently demonstrated that observers are less likely to be captured by abruptly appearing, task-irrelevant stimuli when they perform a search that is high, as opposed to low, in perceptual load (Cosman \& Vecera, 2009), consistent with perceptual load theory. However, recent results indicate that onset frequency can influence stimulus-driven capture, with infrequent onsets capturing attention more often than did frequent onsets. Importantly, in our previous task, an abrupt onset was present on every trial, and consequently, attentional capture might have been affected by both onset frequency and perceptual load. In the present experiment, we examined whether onset frequency influences attentional capture under conditions of high perceptual load. When onsets were presented frequently, we replicated our earlier results; attentional capture by onsets was modulated under conditions of high perceptual load. Importantly, however, when onsets were presented infrequently, we observed robust capture effects. These results conflict with a strong form of load theory and, instead, suggest that exposure to the elements of a task (e.g., abrupt onsets) combines with high perceptual load to modulate attentional capture by task-irrelevant information.
\end{abstract}

Scenes contain a tremendous amount of informationoften, more than an observer can process at one time. As a result, selective attention mechanisms have developed that allow us to focus only on the information most relevant for carrying out our goals. For example, when attempting to read a newspaper in a crowded coffeehouse, we focus on the words on the page and ignore the irrelevant sights and sounds around us. Such goal-directed attentional control allows us to focus on the task at hand without interruption from extraneous information. However, sometimes our attention is captured by salient information in the environment, regardless of its relevance to our goals. This type of stimulus-driven attentional capture is ubiquitous and can cause us to shift away from our primary goals and attend to information outside of our current focus.

In recent years, there has been a great amount of debate regarding the nature of such stimulus-driven attentional capture. Whereas some believe that stimulus-driven control is automatic, occurring independently of our goals (e.g., Theeuwes, 1994), others have posited that attentional capture is under the top-down control of the observer (e.g., Folk, Remington, \& Johnston, 1992). This debate is ongoing, with evidence in favor of both purely stimulusdriven capture (Christ \& Abrams, 2006; Schreij, Owens, $\&$ Theeuwes, 2008), and top-down contingent control over capture (Folk \& Remington, 2006, 2008; Leber \& Egeth, 2006). As a result, there has been a great deal of focus on the particular types of stimuli and stimulus parameters that allow for each mode of control.

One class of stimuli that has been shown to capture attention in a stimulus-driven manner comprises items that appear abruptly within a visual scene (Christ \& Abrams, 2006; Yantis \& Jonides, 1984). For example, when participants are asked to search for a target item in a scene, reaction times (RTs) to the target are shorter when that item is an abruptly appearing object than when it is not (Christ \& Abrams, 2006; Yantis \& Jonides, 1984). This onset capture during search occurs even when onset status does not predict which item will be the target (i.e., the target and distractor items are equally likely to be defined by an abrupt onset), and as a result, some have suggested that abrupt onsets can capture attention in a stimulus-driven manner (Christ \& Abrams, 2006; Schreij et al., 2008). Several other types of visual events also appear to capture attention potently, including motion onsets (Abrams \& Christ, 2003), looming motion (Franconeri \& Simons, 2003), and, perhaps, the offset, or disappearance, of an object (Pratt \& McAuliffe, 2001; but see Cole \& Kuhn, 2010).

However, under some circumstances, these various forms of attentional capture can be attenuated, demonstrating that such capture is not entirely stimulus driven but is under the top-down control of the observer. For example, under some circumstances, onset capture occurs only when participants have an attentional control setting for onset stimuli (Folk et al., 1992; Gibson \& Kelsey, 1998). Also, if a target's location is precued by a $100 \%$ valid cue, an abruptly appearing distractor loses its ability to capture attention (Yantis \& Jonides 1990). Finally, some of our recent work has indicated that onset capture is modulated by the perceptual load of a display (Cosman \& Vecera, 2009). With high-load displays that contained

J. D. Cosman, joshua-cosman@uiowa.edu 
a target and several distractors, we found that salient onsets failed to capture attention. Onset capture occurred only with low-load displays that contained a target but no distractors (see also Cosman \& Vecera, 2010, for similar results regarding attentional capture by motion).

A straightforward interpretation of our results is that abrupt onsets do not capture attention automatically. Instead, in demanding, high-load situations, observers have fewer attentional resources to "spill over" to a taskirrelevant onset distractor, and as a result, onsets do not interfere with search performance. We based our interpretation on Lavie's (1995; Lavie \& Tsal, 1994) load theory, which proposes that perceptual-level attention resources are exhausted when a demanding, high-perceptual-load search is performed. Load theory explains a wide range of attentional phenomena, and our application of load theory to onset capture indicates that perceptual load is a critical factor in attentional capture.

Despite the foregoing evidence suggesting that onset capture can be abolished under certain conditions, recent findings have demonstrated that presentation frequency affects the ability of abruptly appearing stimuli to capture attention. Neo and Chua (2006) reexamined the claim that observers can override onset capture when target location is known in advance. Using a cuing paradigm, Neo and Chua showed that the frequency of an onset distractor item affects attentional capture by that distractor. Task-irrelevant onset distractors were presented either frequently ( $75 \%$ of the trials) or infrequently $(18.75 \%$ of the trials) at a nontarget location, and target location was $100 \%$ validly cued prior to the presentation of the onset distractor. When task-irrelevant onsets were presented frequently, onsets lost their ability to capture attention, replicating previous findings (e.g., Theeuwes, 1991; Yantis \& Jonides, 1990). However, when task-irrelevant onsets were presented infrequently, the onset distractor produced significant interference with target identification, indicating that the onsets retained their ability to capture attention. On the basis of these results, the authors suggested that to capture attention, abrupt onsets need to be "novel" (i.e., infrequent) and that top-down control over onset capture might occur only when onsets appear frequently (for related effects of novelty on capture by color singletons, see also Horstmann, 2002; Horstmann \& Ansorge, 2006).

One direct implication of Neo and Chua's (2006) findings is that onset frequency, not perceptual load alone, may determine attentional capture. In our previous work, an abruptly appearing flanker appeared on every trial, and it is therefore possible that these frequent onset distractors gave participants sufficient exposure to effectively control onset capture during high-load search. In the present experiment, we tested between a frequency account of onset capture and a load-modulated account of capture.

Here, we used a paradigm similar to that in Cosman and Vecera (2009), but we varied the frequency with which onset distractors appeared. Participants searched high-load displays for a known target among visually similar distractors. A task-irrelevant flanking distractor appeared either above or below the search array, and the flanker appeared as either an onset or an offset. The flanker's identity was either incompatible or compatible with respect to the target, allowing us to measure the extent to which the flanker interfered with responses to the target. Typically, no response interference occurs in high-load displays, because attentional resources are exhausted by the search task and are unable to be allocated to the flanker (Lavie, 1995).

To distinguish a frequency account of capture from the predictions made by load theory, we manipulated the frequency of the onset flankers. In the $80 \%$ onset condition, an onset flanker appeared frequently ( $80 \%$ of the trials), and the offset flanker appeared infrequently $(20 \%$ of the trials). In the $20 \%$ onset condition, we switched the frequency of the distractors such that the flanker now appeared as an onset infrequently ( $20 \%$ of the trials) and an offset frequently ( $80 \%$ of the trials).

Load theory would predict no effect of our frequency manipulation on onset capture, because all the displays involved high perceptual load; thus, our displays should exhaust the capacity of perceptual-level attention, and onset distractors should not interfere with performance, regardless of their frequency. In contrast, a frequency account predicts that experience with onset flankers will determine attentional capture. Consequently, frequently appearing onsets in the $80 \%$ onset condition should fail to capture attention. But, importantly, the infrequently appearing onsets in the $20 \%$ onset condition should capture attention, despite the high-perceptual-load displays.

\section{METHOD}

\section{Participants}

Twenty-eight University of Iowa undergraduates participated for course credit, 14 in the $80 \%$ onset condition and 14 in the $20 \%$ onset condition. All had normal or corrected-to-normal vision.

\section{Stimuli and Procedure}

A Macintosh minicomputer displayed stimuli on a 17 -in. CRT monitor and recorded responses and response latencies. The experiment was controlled using MATLAB and the Psychophysics Toolbox (Brainard, 1997).

Aside from onset/offset frequency, the stimuli and procedure were identical for the $80 \%$ onset and $20 \%$ onset conditions. The observers sat $75 \mathrm{~cm}$ from the monitor in a dimly lit room. We used a task nearly identical to that in the high-load condition in Cosman and Vecera (2009). A sample trial appears in Figure 1. A fixation point measuring $0.35^{\circ} \times 0.35^{\circ}$ appeared for $1,000 \mathrm{msec}$, followed by a placeholder array for $1,000 \mathrm{msec}$. The placeholder display contained a central array of six figure-eight placeholders subtending $7.50^{\circ} \times$ $1.60^{\circ}$ of visual angle, with each placeholder measuring $1.50^{\circ} \times$ $0.75^{\circ}$, with a distance of $0.45^{\circ}$ between each placeholder. The placeholder for the offset flanker $\left(1.90^{\circ} \times 0.90^{\circ}\right)$ appeared either above or below the six centrally located placeholders. The center of the flanking placeholder (and the flanker letter itself in both onset and offset flanker conditions) was positioned $3.0^{\circ}$ from the fixation point and $4.5^{\circ}$ from the center of the most eccentric placeholders in the central array.

Next, line segments disappeared from the placeholders. Simultaneously with the offset of the line segments, a flanker letter appeared either in the position of the flanker placeholder (i.e., segments were removed from the flanker placeholder to generate an offset flanker) or opposite this placeholder (i.e., a new object appeared as an onset flanker). Flankers were either compatible or incompatible with re- 


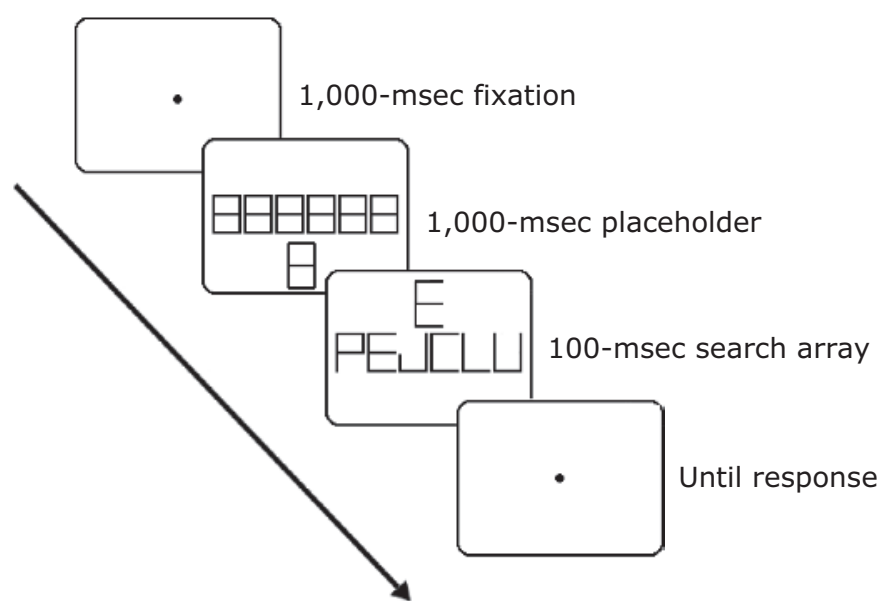

Figure 1. Sequence of events and timing parameters for the task used in both conditions. Following presentation of a fixation point for $1,000 \mathrm{msec}$, a six-item placeholder array flanked by a cortically scaled flanker placeholder was presented for $1,000 \mathrm{msec}$. Directly after this, a high-load search array and a single flanker item (onset or offset) appeared for $100 \mathrm{msec}$. In the onset frequent condition, the frequency of onset flankers was held at $80 \%(20 \%$ offset flankers), whereas in the onset infrequent condition, the frequency of onset flankers was decreased to $20 \%(80 \%$ offset flankers). The trial depicted is a congruent onset flanker trial.

spect to the target on a given trial. The resulting high-load displays contained a single flanker letter and six centrally located, taskrelevant letters containing a target and five distractors. This search array and flanker item remained visible for $100 \mathrm{msec}$, too brief a duration to permit eye movements during search. The observers' task was to report the identity of a target letter, which was either an $\mathrm{E}$ or an $\mathrm{H}$ embedded within an array of the five distractor letters $\mathrm{U}, \mathrm{L}, \mathrm{P}, \mathrm{C}$, or J. Each letter was equally likely to appear in any of the six different positions in the search array.

The flanker letter was either an $\mathrm{E}$ or an $\mathrm{H}$ that was either compatible or incompatible with the target letter on a given trial. The observers reported the target's identity by pressing either the " $\mathrm{z}$ " or the "/" key, with response keys for the E and $\mathrm{H}$ targets being counterbalanced between observers. Following 48 practice trials, observers responded to 288 experimental trials. Each block contained 48 trials, and the participants completed six blocks of trials. We informed the observers that the flanker letters were not relevant to the task and stressed that they should be ignored. We also instructed the observers to maintain fixation throughout the duration of the experiment. With this design, it was possible to examine the effects of task-irrelevant onset and offset flankers, presented with varying frequency, on search performance under conditions of high perceptual load.

\section{RESULTS}

Only correct trials were analyzed. In both conditions, RTs less than $150 \mathrm{msec}$ or greater than $2,500 \mathrm{msec}$ were excluded from the analyses; this trimming eliminated less than $2 \%$ of the data in both the $80 \%$ onset and $20 \%$ onset conditions. The observers' mean correct RT and error rate data for each condition are shown in Figure 2. A combined analysis was performed to test for differences in capture across the two frequency conditions. RT data from both experiments were entered into a $2 \times 2 \times 2$ mixed ANOVA, with onset frequency ( $80 \%$ onset vs. $20 \%$ onset) as a between-subjects factor and onset status (onset vs. offset) and congruency (compatible vs. incompatible) as within-subjects factors. Of primary interest in this analysis was the interaction between onset capture and presentation frequency. The combined analysis yielded significant two-way interactions between onset frequency and onset status $[F(1,24)=21.8, p<.01]$ and between onset frequency and congruency $[F(1,24)=6.7, p<.05]$. Importantly, the three-way interaction between onset frequency, onset status, and congruency was significant $[F(1,24)=$ $4.9, p<.05$ ], indicating that the ability of onsets to capture attention and drive a flanker effect depended on the frequency with which the onsets were presented.

Both accuracy and RT data for each frequency condition were also analyzed individually with separate $2 \times 2$ repeated measures ANOVAs, with flanker type (onset vs. offset) and flanker congruency (compatible vs. incompatible) as factors. For the $80 \%$ onset condition, no main effects or interactions were significant $[F \mathrm{~s}(1,13)<0.05$, $p$ s $>$.83]. This indicates that RTs in the onset flanker condition were similar between compatible $(732 \mathrm{msec})$ and incompatible $(725 \mathrm{msec})$ flankers, RTs in the offset flanker condition were similar between compatible (723 msec) and incompatible (728 msec) flankers, and the overall RTs between onset $(729 \mathrm{msec})$ and offset $(726 \mathrm{msec})$ conditions were similar. Furthermore, no main effects or interactions were observed for the accuracy data $(F \mathrm{~s}<0.6, p \mathrm{~s}>.46)$. These findings replicate the effect of perceptual load on onset capture observed in Cosman and Vecera (2009) and suggest that when onsets are presented frequently during high-load search tasks, observers can effectively attenuate distraction by taskirrelevant onsets. 


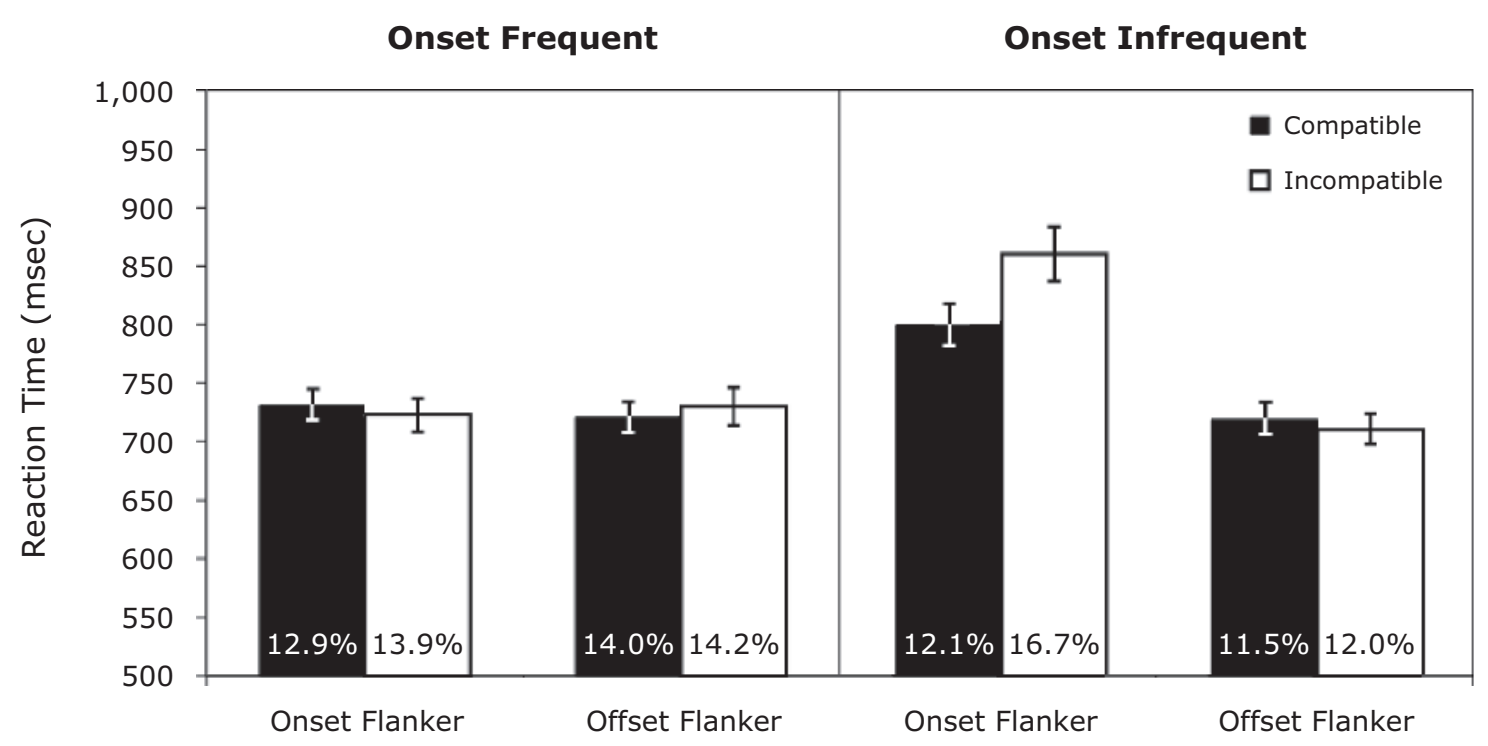

Figure 2. Mean reaction times for high-load search when flankers were compatible or incompatible onsets or offsets in the onset frequent condition (left panel) and the onset infrequent condition (right panel). Error rates for each condition are indicated at the base of the graph. Error bars represent $95 \%$ within-subjects confidence intervals for the flanker effect in each condition (Loftus \& Masson, 1994).

In contrast to the $80 \%$ onset condition, analysis of the data from the $20 \%$ onset condition revealed a main effect of onset status on $\mathrm{RTs}[F(1,13)=7.8, p<.01]$, where RTs on trials in which the flanker was an onset were significantly longer $(828 \mathrm{msec})$ than those on trials in which the flanker was an offset $(721 \mathrm{msec})$. In addition, there was a main effect of congruency $[F(1,13)=11.3, p<.01]$, and the interaction between onset status and congruency was also significant $[F(1,13)=15.8, p<.01]$. Planned comparisons revealed that this interaction was driven by a significant flanker effect in the onset condition, where RTs on trials in which the flanker was incompatible were significantly longer $(859 \mathrm{msec})$ than those on trials in which the flanker was compatible $(796 \mathrm{msec})[t(13)=4.7, p<$ .01]. Error rates did not differ significantly between onset and offset flanker conditions, although there was a trend toward higher error rates in the onset flanker condition $[F(1,13)=4.3, p<.06]$, but error rates did differ between compatible and incompatible flanker conditions $[F(1,13)=9.4, p<.01]$. The interaction between onset status and congruency was also significant $[F(1,13)=$ $15.8, p<.01]$.

\section{DISCUSSION}

Our results indicate that the frequency with which an onset appears affects attentional capture in highperceptual-load search displays. Frequently presented onsets failed to capture attention when participants searched through high-perceptual-load displays, replicating Cosman and Vecera (2009). Perhaps more important, our present results indicated that infrequently occurring onset flankers captured attention, even though participants were performing a high-load search. These results suggest that frequency and high perceptual load combine to determine whether or not capture will occur, supporting a frequency account of capture (Neo \& Chua, 2006) but challenging load theory (Lavie, 1995).

Although our results have implications both for theories of attentional capture and for theories of perceptual load, one potential concern is our use of compatible and incompatible flankers. Given that load effects are often calculated as incompatible-flanker- minus neutral-flanker-trial RTs (Lavie, 1995; Lavie, Hirst, de Fockert, \& Viding, 2004) and, furthermore, it has been demonstrated that the use of incongruent minus congruent RTs in assessing load effects tends to underestimate flanker interference (see Biggs \& Gibson, 2010), it is possible that the present results may have been influenced by our measure of flanker interference, and not by onset frequency. To exclude this possibility, we conducted a supplementary experiment with 24 new participants (12 in each frequency condition) that was identical to that presented above, except that the flankers were either neutral or incompatible. The results from this supplementary experiment are shown in Figure 3 , and the findings replicated the results of our main experiment. This indicates that our conclusions hold irrespective of the types of flankers used.

We should note that there are two possible measures of onset capture in our experiment. ${ }^{1}$ First, the difference between compatible and incompatible flankers provides a measure of flanker processing (i.e., identification), and flanker identification was clearly abolished for frequent onset flankers, but not for infrequent onset flankers. Second, in the infrequent onset condition, the onsets caused a general lengthening of RTs, regardless of their compatibility. This finding would seem to suggest that under high-load conditions, attentional capture 


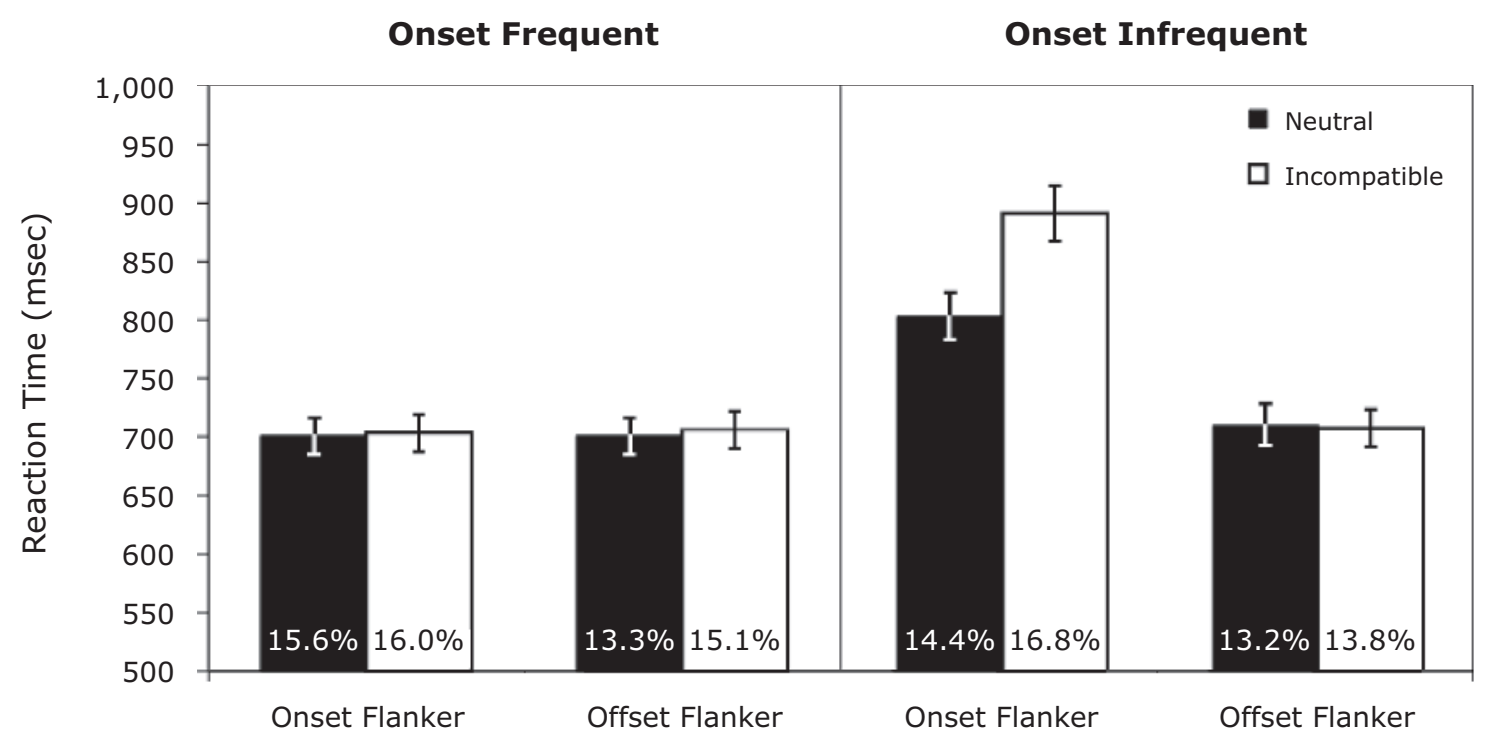

Figure 3. Mean reaction times for high-load search when flankers were incompatible or neutral onsets or offsets, in a supplementary experiment using incompatible and neutral flanker letters (see the Discussion section). The methods employed in this experiment were identical to those in Experiment 1, except for the use of incompatible and neutral flankers. Error rates for each condition are indicated at the base of the graph. Error bars represent $95 \%$ within-subjects confidence intervals for the flanker effect in each condition

by frequent onsets is initially automatic but is attenuated with sufficient experience, given that a similar lengthening of RTs was absent in the high-load, frequent onset condition. Taken together with the results of our previous work showing that even frequently presented onsets cause interference in displays that are low in perceptual load (Cosman \& Vecera, 2009), it appears that capture by abrupt onsets may proceed automatically unless displays are sufficiently high in perceptual load and abrupt onsets are presented frequently. Such an interpretation is tentative, however, given that in the present experiment and in our previous work, our primary assay of capture was the presence or absence of a flanker effect, and not overall RTs in each condition.

Regardless of the nature of the effect of load on capture by onsets, these results challenge perceptual load theory, because perceptual load effects are typically described as a resource limitation in perceptual-level attention: To the extent that perceptual resources are exhausted by the demands of the primary task, there will be no resources left over to process task-irrelevant visual information (Lavie, 1995; Lavie et al., 2004). Given the results of the present study, it would seem that perceptual load effects reflect the combination of higher level attentional control settings (e.g., Folk et al., 1992; Neo \& Chua, 2006; Theeuwes, Kramer, \& Belopolsky, 2004) and lower level perceptual effects, such as the competition between items in a search array (e.g., Torralbo \& Beck, 2008). Thus, future conceptualizations of perceptual load theory must account for the effects of nonperceptual, top-down control mechanisms that appear to influence distractor interference.

In line with this interpretation, other recent results show that load effects depend on prior experience with a task
(Couperus, 2009; Theeuwes et al., 2004). For example, Theeuwes et al. showed that intermixing high- and lowperceptual-load trials affected capture. When observers performed a high-load search on the current trial, a taskirrelevant distractor produced an interference effect if the previous trial had been low in perceptual load. They explained their result in terms of the scale of attention, suggesting that participants configured the size of their attentional "window" on a trial-by-trial basis. On low-load trials, participants adopted a wide scale of attention, such that when the subsequent trial was high in perceptual load the peripheral distractor was processed.

Likewise, Couperus (2009) showed, using ERPs, that the visual cortical response to parafoveally presented, task-irrelevant probe items is modulated by learned expectancies regarding the load of the primary task. If the trial type (high vs. low load) could be predicted on the basis of learned expectancies (i.e., sequences of particular trial types), there was a diminished $\mathrm{P} 1$ response to a taskirrelevant probe item on high-load trials and an increased P1 response on low-load trials - what may be considered a "typical" load effect. However, when the trial type was random with respect to load, such an effect was not observed. This effect was interpreted in the context of the results of Theeuwes et al. (2004), and it was suggested that the anticipation of particular types of stimulus arrays (high vs. low load) can lead to changes in attentional scale and, thus, the extent of distractor processing.

Taking these findings together with the present results, it appears that task experience combines with high perceptual load to modulate the processing of task-irrelevant information, with a critical factor being the frequency with which the task-irrelevant attributes appear. This sug- 
gests that efficient suppression of task-irrelevant information reflects the combined influences of bottom-up and top-down processes and that future conceptualizations of perceptual load theory must take top-down control processes into account when explaining the effects of load on attentional selection.

\section{AUTHOR NOTE}

This research was supported in part by a grant from the National Science Foundation (BCS 03-39171) to S.P.V. We thank Ed Awh, Fook Chua, Clayton Hickey, and anonymous reviewers for their useful input regarding an earlier version of the manuscript. Correspondence should be addressed to J. D. Cosman, Departments of Neuroscience and Psychology, University of Iowa, E305 Seashore Hall, Iowa City, IA 522421407 (e-mail: joshua-cosman@uiowa.edu).

Note-Accepted by Cathleen M. Moore's editorial team.

\section{REFERENCES}

Abrams, R. A., \& Christ, S. E. (2003). Motion onset captures attention. Psychological Science, 14, 427-432.

Biggs, A. T., \& Gibson, B. S. (2010). Competition between color salience and perceptual load during visual selection can be biased by top-down set. Attention, Perception, \& Psychophysics, 72, 53-64.

Brainard, D. H. (1997). The Psychophysics Toolbox. Spatial Vision, 10, 433-436.

Christ, S. E., \& Abrams, R. A. (2006). Abrupt onsets cannot be ignored. Psychonomic Bulletin \& Review, 13, 875-880.

Cole, G. G., \& Kunn, G. (2010). Attentional capture by object appearance and disappearance. Quarterly Journal of Experimental Psychology, 63, 147-159.

Cosman, J. D., \& Vecera, S. P. (2009). Perceptual load modulates attentional capture by abrupt onsets. Psychonomic Bulletin \& Review, 16, 404-410.

Cosman, J. D., \& Vecera, S. P. (2010). Attentional capture by motion onsets is modulated by perceptual load. Attention, Perception, \& Psychophysics, 72, 2096-2105.

COUPERUS, J. W. (2009). Implicit learning modulates selective attention at sensory levels of perceptual processing. Attention, Perception, \& Psychophysics, 71, 342-351.

FolK, C. [L.], \& REMINGTON, R. [W.] (2006). Top-down modulation of preattentive processing: Testing the recovery account of contingent capture. Visual Cognition, 14, 445-465.

Folk, C. L., \& Remington, R. W. (2008). Bottom-up priming of topdown attentional control settings. Visual Cognition, 16, 215-231.

Folk, C. L., Remington, R. W., \& Johnston, J. C. (1992). Involuntary covert orienting is contingent on attentional control settings. Journal of Experimental Psychology: Human Perception \& Performance, 18 , 1030-1044.

Franconeri, S. L., \& Simons, D. J. (2003). Moving and looming stimuli capture attention. Perception \& Psychophysics, 65, 999-1010.

Gibson, B. S., \& Kelsey, E. M. (1998). Stimulus-driven attentional capture is contingent on attentional set for displaywide visual features. Journal of Experimental Psychology: Human Perception \& Performance, 24, 699-706.

HorstMAnN, G. (2002). Evidence for attentional capture by a surprising color singleton in visual search. Psychological Science, 13, 499-505.

Horstmann, G., \& Ansorge, U. (2006). Attentional capture by rare singletons. Visual Cognition, 14, 295-325.

Lavie, N. (1995). Perceptual load as a necessary condition for selective attention. Journal of Experimental Psychology: Human Perception \& Performance, 21, 451-468.

Lavie, N., Hirst, A., De Fockert, J. W., \& Viding, E. (2004). Load theory of selective attention and cognitive control. Journal of Experimental Psychology: General, 133, 339-354.

Lavie, N., \& Tsal, Y. (1994). Perceptual load as a major determinant of the locus of selection in visual attention. Perception \& Psychophysics, 56, 183-197.

Leber, A. B., \& Egeth, H. E. (2006). It's under control: Top-down search strategies can override attentional capture. Psychonomic Bulletin \& Review, 13, 132-138.

Loftus, G. R., \& Masson, M. E. J. (1994). Using confidence intervals in within-subject designs. Psychonomic Bulletin \& Review, 1, 476-490.

Neo, G., \& ChuA, F. K. (2006). Capturing focused attention. Perception \& Psychophysics, 68, 1286-1296.

Pratt, J., \& McAuliffe, J. (2001). The effects of onsets and offsets on visual attention. Psychological Research, 65, 185-191.

SchreiJ, D., Owens, C., \& Theeuwes, J. (2008). Abrupt onsets capture attention independent of top-down control settings. Perception \& Psychophysics, 70, 208-218.

TheEuwes, J. (1991). Exogenous and endogenous control of attention: The effect of visual onsets and offsets. Perception \& Psychophysics, 49, 83-90.

TheEuwes, J. (1994). Stimulus-driven capture and attentional set: Selective search for color and abrupt onsets. Journal of Experimental Psychology: Human Perception \& Performance, 20, 799-806.

Theeuwes, J., Kramer, A. F., \& Belopolsky, A. V. (2004). Attentional set interacts with perceptual load in visual search. Psychonomic Bulletin \& Review, 11, 697-702.

Torralbo, A., \& BeCK, D. M. (2008). Perceptual-load-induced selection as a result of local competitive interactions in visual cortex. Psychological Science, 19, 1045-1050.

YAnTIS, S., \& Jonides, J. (1984). Abrupt visual onsets and selective attention: Evidence from visual search. Journal of Experimental Psychology: Human Perception \& Performance, 10, 601-621.

Yantis, S., \& Jonides, J. (1990). Abrupt visual onsets and selective attention: Voluntary versus automatic allocation. Journal of Experimental Psychology: Human Perception \& Performance, 16, 121134.

\section{NOTE}

1. We thank Fook Chua for this suggestion.

(Manuscript received January 15, 2010; revision accepted for publication April 14, 2010.) 